\title{
Identifying significant pathways of hepatitis B virus-related hepatocellular carcinoma based on crosstalk and network pathways
}

\author{
L.M. Wen ${ }^{1}$, W.Z. Wu ${ }^{2}$ and X.C. Peng ${ }^{3}$ \\ ${ }^{1}$ Department of Hepatobiliary, Pancreatic and Splenic Surgery, Clinical College, \\ Inner Mongolia People's Hospital, Inner Mongolia Medical University, \\ Hohhot City, Inner Mongolia, China \\ ${ }^{2}$ Department of General Surgery, \\ Ningbo Medical Treatment Center Lihuili Hospital (East), Ningbo, \\ Zhejiang Province, China \\ ${ }^{3}$ Department of Oncology, Modern Hospital of Guangzhou, Guangzhou, China \\ Corresponding author: W.Z. Wu \\ E-mail: weizhuwu2015@sina.com
}

Genet. Mol. Res. 15 (2): gmr. 15028343

Received December 28, 2015

Accepted February 11, 2016

Published June 24, 2016

DOI http://dx.doi.org/10.4238/gmr.15028343

\begin{abstract}
This study aims to identify significant pathways in hepatitis B virus (HBV)-related hepatocellular carcinoma (HCC) based on the pathway network strategy. We proposed a pathway network where a protein-protein interaction (PPI) network was integrated with the crosstalk of pathways. Pathway data were first obtained from background PPI network, Reactome pathway database, and common genes between mRNA differentially expressed genes (DEGs), and miRNA target genes of HBV-related HCC. Pathway interactions were subsequently randomly extracted based on gene-gene interactions, and a weight value was assigned to each crosstalk using the Spearman correlation coefficient. Finally, pathways and crosstalk were visualized via Cytoscape to construct the final pathway network. A total of 9 common genes were identified between 396 mRNA DEGs and
\end{abstract}


400 miRNA target genes, and 17 pathways were identified based on background pathways and common genes. In addition, we constructed a pathway network that included 136 interactions and 17 pathways. The weight value of netrin-1 signaling and regulation of Frizzled proteins (FZD) by ubiquitination was the largest, at 0.228 . In conclusion, we identified 17 significant pathways that might act as potential biomarkers of HBV-related HCC. This information may offer some insight into treatment and detection of HBV-related HCC.

Key words: Hepatocellular carcinoma; Hepatitis B virus; Pathway; Network; Crosstalk

\section{INTRODUCTION}

Hepatocellular carcinoma (HCC) is the fifth most common cancer worldwide, and is the third leading cause of cancer-related mortality (Kaseb et al., 2013). Most cases of HCC (approximately 80\%) are associated with chronic hepatitis B virus (HBV) or hepatitis $\mathrm{C}$ virus infections (Aoki et al., 2014). However, variations in age, gender, race, geographic regions, timing of the viral infection, as well as age of affected individuals all contribute to differences in disease prevalence and progression of viral hepatitis and HCC (El-Serag, 2012). In addition, due to complexity of disease, gene expression profiles have become an attractive area of research for HCC.

Based on gene expression profile studies, HCC has been linked to activation of epidermal growth factor receptor (EGFR), insulin-like growth factor receptor (IGFR), WNT$\beta$-catenin, and the PI3K-AKT-mTOR signaling pathways (Pan et al., 2007; Llovet and Bruix, 2008; Jiang et al., 2011; Diao et al., 2012). However, early biomarkers and tumor-specific treatments for HCC are still lacking. Some studies showing that several signaling cascades are activated in the liver before the appearance of tumors suggest that pathway addiction may be important to the pathogenesis of HCC (Arzumanyan et al., 2013).

Therefore, the objective of this study was to identify significant pathways in HBV-related HCC based on pathway network strategy and crosstalk. To realize this, pathway data were obtained from background protein-protein interaction (PPI) network, Reactome pathway database, and common genes between mRNA differentially expressed genes (DEGs), and miRNA target genes of HCC. Next, pathway interactions were randomly extracted from gene-gene interactions, and a weight value was assigned to each crosstalk based on the absolute difference of Spearman correlation coefficient (SCC) under diseased and normal condition. Finally, pathways and interactions were visualized by Cytoscape for construction of pathway network.

\section{MATERIAL AND METHODS}

\section{Gene data}

Genome-wide expression profiles of both miRNAs and mRNAs from tumor and adjacent non-tumor tissues in HBV-related HCC patients (E-GEOD-22058) (Burchard et al., 2010) were collected from the publicly available database ArrayExpress. Microassay technology was performed using a customized cDNA platforms, A-GEOD-6793-Rosetta/ 
Merck Human RSTA Custom Affymetrix 1.0 microarray Platform and A-GEOD-10457Rosetta human miRNA qPCR array Platform. Analysis was carried out on 197 mRNA samples (97 normal controls and 100 HBV-related HCC samples) and 192 miRNA samples (96 normal controls and $96 \mathrm{HBV}$-related HCC samples). After downloading the gene annotation files and mapping the IDs of mRNA probes to gene symbols, we obtained a gene expression dataset with 11,525 genes for mRNA and 220 genes for miRNA-related analysis.

\section{Identifying DEGs for mRNA}

In the present paper, we applied SAM to determine gene expression changes between controls and HBV-related HCC patients. SAM identifies genes with statistically significant changes in expression by assimilating a set of gene-specific $t$-tests (Li and Tibshirani, 2013). A statistic based on the ratio of change in gene expression to standard deviation for that gene, the relative difference $d(i)$, was calculated to account for gene-specific fluctuations. Subsequently, genes were ranked in descending order of $d(i)$ values, where $d(1)$ was the largest relative difference, $d(2)$ was the second largest relative difference, and $d(i)$ was the $i$ th largest relative difference. The expected relative difference, $d_{E}(i)$, was defined as the average of all permutations:

$$
d_{E}(i)=\frac{\sum_{t} d_{t}(i)}{n}
$$

where $d_{t}(i)$ was the $i$ th largest relative difference for permutation $t$. For the vast majority of genes, $d(i) \cong d_{E}(i)$, but some genes were represented by points displaced from the $d(i)=d_{E}(i)$ line by a distance greater than the threshold $\Delta$. As $\Delta$ decreased, the number of significant genes as calculated by SAM increased. We set $\Delta=10.30$ for DEGs identification of HBV-related HCC mRNA.

\section{Predicating target genes for miRNA}

Similarly, SAM was selected to identify differential miRNAs between control and HBV-related HCC miRNA samples with a threshold of $\Delta=6.06$. We gained 11 differential miRNAs and conducted miRNA target predictions using the online tool miRWalk 2.0 (http:// www.umm.uni-heidelberg.de/apps/zmf/mirwalk/), a comprehensive atlas of predicted and validated miRNA-target interactions (Dweep et al., 2011; Dweep and Gretz, 2015). The miRWalk algorithm, a target gene prediction method, was based on a computational approach written in Perl programming language to identify multiple consecutive Watson-Crick complementary subsequences between miRNA and gene sequences (Dweep et al., 2011). This approach is an integration of four other methods of predicating target genes (miRanda, miRDB, RNA22, and Targetscan). Genes with SUM $=5$ (miRanda, miRDB, miRWalk, RNA22, and Targetscan) were denoted as target genes for miRNA. By cross-referencing with the DEGs of mRNA data, we were able to determine common genes that may play significant roles in the progression of HBV-related HCC (Figure 1A). 

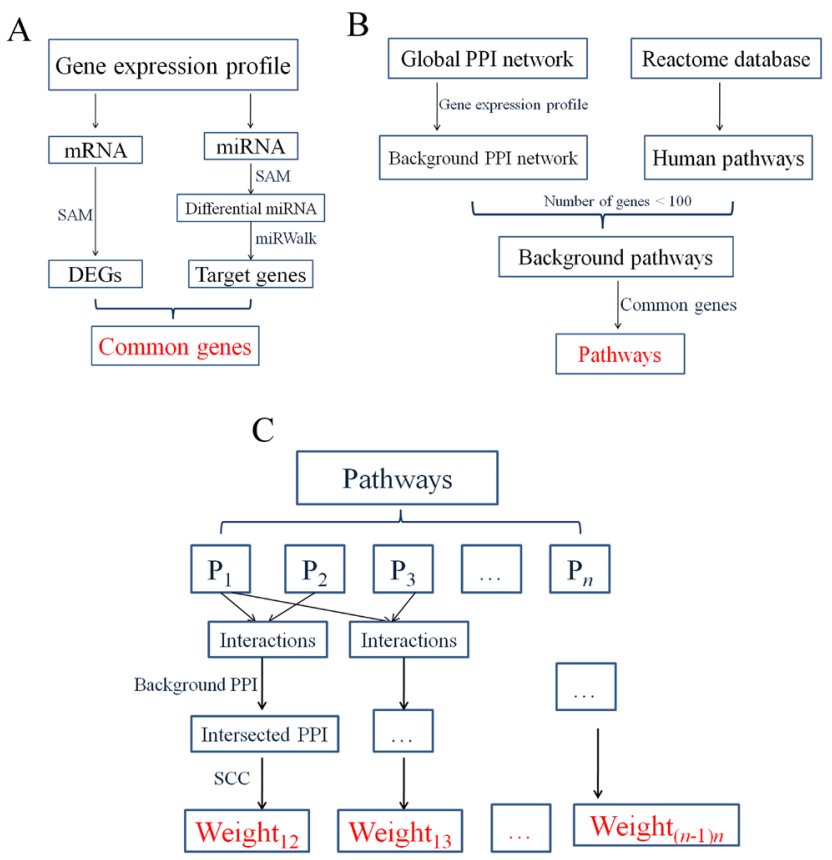

Figure 1. Study design schematics of (A) finding common genes, (B) obtaining pathway data, and (C) establishing weighted values for pathway interactions.

\section{Pathway data}

Global PPI interactions were downloaded from the Search Tool for the Retrieval of Interacting Genes/Proteins (STRING) database, which included 787,896 interactions in humans. By integrating the global PPI network and the gene expression profile E-GEOD-22058, we extracted two interaction nodes in the expression profile.

A total of 1675 human pathways were downloaded from Reactome pathway database, an online curated resource for human pathway data that provides infrastructure for computation across the biologic reaction network (Croft et al., 2011). We calculated the number of interacting genes between each pathway and background PPI networks. As pathways with a small number of genes are more easily investigated, pathways with a gene set $>100$ were filtered out (Ahn et al., 2014). We then selected pathways that included common genes to carry out further analysis (Figure 1B).

\section{Crosstalk of pathways}

In order to evaluate the interactions between pathways, a weight value was assigned to any two pathways (Figure 1C). If weight $>0$, it was defined as crosstalk between the pathways. We first randomly constructed interactions between genes in the pathways. The SCC (Myers and Sirois, 2006) was then determined for intersected crosstalk in two pathways using the absolute differences between HBV-related HCC and normal controls. The mean value of absolute difference for all intersected interactions was denoted as the weight between two 
pathways. Using this analogy, we could obtain all interactions and their weight values for any two pathways. For example, in pathway 1, genes enriched in pathway 1 and pathway 2 were first used to build gene interaction. By integrating these interactions with background PPI network, the intersections were considered as background pathway interactions between pathway 1 and pathway 2. Subsequently, the pathway intersections under HBV-related HCC and normal conditions were weighted using SCC. The SCC of a pair of interactions ( $x$ and $y$ ) was defined as:

$$
S C C(x, y)=\frac{1}{s-1} \sum_{i=1}^{s}\left(\frac{g(x, i)-\bar{g}(x)}{\sigma(x)}\right) \cdot\left(\frac{g(y, i)-\bar{g}(y)}{\sigma(y)}\right)
$$

(Equation 2)

where $s$ is the number of interactions; $g(x, i)$ or $g(y, i)$ is the expression level of interaction $x$ or $y$ in the pathway $i$ under a specific condition (HBV-related HCC or normal); $\bar{g}(x)$ or $\bar{g}(y)$ represent the mean expression level of interaction $x$ or $y$, and $s(x)$ or $\mathrm{s}(y)$ represent the standard deviation of expression level of interaction $x$ (or $y$ ). By calculating the absolute difference in interactions between HBV-related HCC and normal conditions, we defined the mean value of absolute differences of all interactions as the weight value between pathway 1 and pathway 2. If no intersection between pathway 1 and pathway 2 were found, the two pathways had no crosstalk, and achieved a weight score of 0 .

\section{Pathway network and analysis}

Pathway network was visualized by Cytoscape (Smoot et al., 2011) based on pathway crosstalk and weight values. To further investigate the functions and significance of specific pathways in the network, we characterized the biological importance of pathways using indices of topological analysis. Quantifying centrality and connectivity helped identify portions of the network that may play interesting roles. Researchers have revealed that topological centrality is effective for identifying essential nodes in well-characterized interaction networks (Prifti et al., 2010). In this paper, topological analyses consisting of degree (Haythornthwaite, 1996), betweenness (Abbasi et al., 2012), closeness (Scott, 2012), stress (Scardoni and Laudanna, 2012), and centrality were conducted for pathway networks.

\section{RESULTS}

\section{Common genes}

In this study, we identified 396 DEGs based on SAM with $\Delta=10.30$ for mRNA. We also found 11 differentially expressed miRNA, of which 5 were up-regulated and 6 were down-regulated. By utilizing miRWalk 2.0, 400 target genes (258 up-regulated and 142 downregulated) were predicted in total. Interestingly, there were 9 common genes between mRNA DEGs and target genes miRNAs: PBK, POLQ, ZBTB9, KPNA2, RSPO3, NTN4, PDGFRA, TMEM154, and CBLN2.

\section{Pathway data}

We downloaded 787,896 human PPIs from the STRING database. From the gene expression profile E-GEOD-22058, 284,111 interactions involving 10,149 genes were 
evaluated, and we denoted this network as background PPI network. A total of 1675 pathways were recruited from the Reactome pathway database. By taking intersections between background PPI network and filtrating pathways, 1505 background pathways were detected. Common genes between mRNA DEGs and miRNA target genes may be more significant than other genes, therefore we intersected background PPI network with common genes and obtained 17 pathways (Table 1) for further analysis. Interestingly, 9 of the 17 pathways were associated with PI3K, especially the PI-3K cascade and the PI3K/AKT pathways. In addition, 7 were signaling related pathways, for example, constitutive PI3K signaling in cancer and PIP3-induced AKT signaling.

Table 1. Common pathways based on common genes between mRNA differentially expressed genes (DEGs) and miRNA target genes.

\begin{tabular}{l|l}
\hline ID & Pathway \\
\hline 1 & Antiviral mechanism by IFN-stimulated genes \\
\hline 2 & Constitutive signaling by aberrant PI3K in cancer \\
\hline 3 & GAB1 signalosome \\
\hline 4 & ISG15 antiviral mechanism \\
\hline 6 & Netrin-1 signaling \\
\hline 7 & Non-integrin membrane-ECM interactions \\
\hline 8 & PI-3K cascade:FGFR1 \\
\hline 9 & PI-3K cascade:FGFR2 \\
\hline 10 & PI-3K cascade:FGFR3 \\
\hline 12 & PI-3K cascade:FGFR4 \\
\hline 13 & PI3K events in ERBB2 signaling \\
\hline 14 & PI3K events in ERBB4 signaling \\
\hline 15 & PI3K/AKT activation \\
\hline 16 & PI3K/AKT signaling in cancer \\
\hline 17 & PIP3 activates AKT signaling \\
\hline
\end{tabular}

\section{Pathway network}

In this paper, we employed crosstalk concept to explore the interactions among 17 pathways. We identified 136 pathway interactions, and the weighted value distribution was illustrated in Figure 2. We found that weight values of a majority of the crosstalk ranged from 0.05-0.15, only one crosstalk was found to have a score of 0.15-0.20 and 0.200.25 . When inputting these interactions into the Cytoscape software, a pathway network with 17 nodes and 136 edges were visualized (Figure 3). Nodes represented pathways, and edges represented crosstalk: the deeper the color, the larger the weighted value. The most changed crosstalk was the pathway interaction between 5 (netrin-1 signaling) and 16 (regulation of FZD by ubiquitination), with a weighted value of 0.228 , and next were 2 (constitutive signaling by aberrant PI3K in cancer) to 16, 7 (PI-3K cascade: FGFR1) to 16, 8 (PI-3K cascade: FGFR2) to 16, and 9 (PI-3K cascade: FGFR3) to 16. The top five crosstalk all included pathway 16 (regulation of FZD by ubiquitination), suggesting that it may play key roles in HBV-related HCC. 


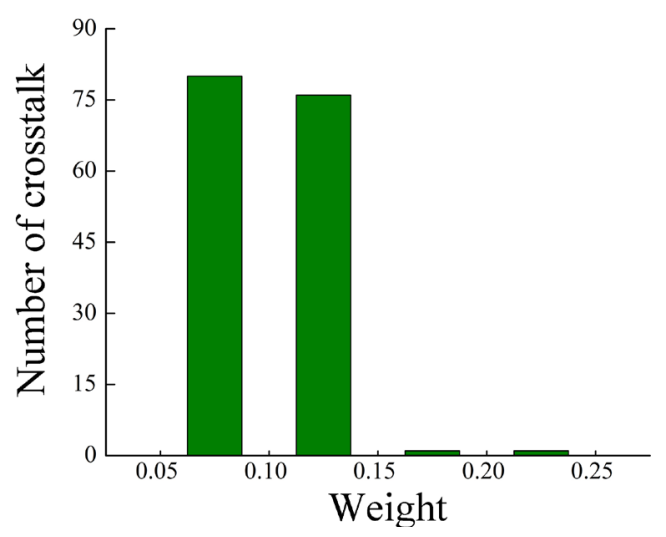

Figure 2. Weight distribution of 136 crosstalks among pathways.

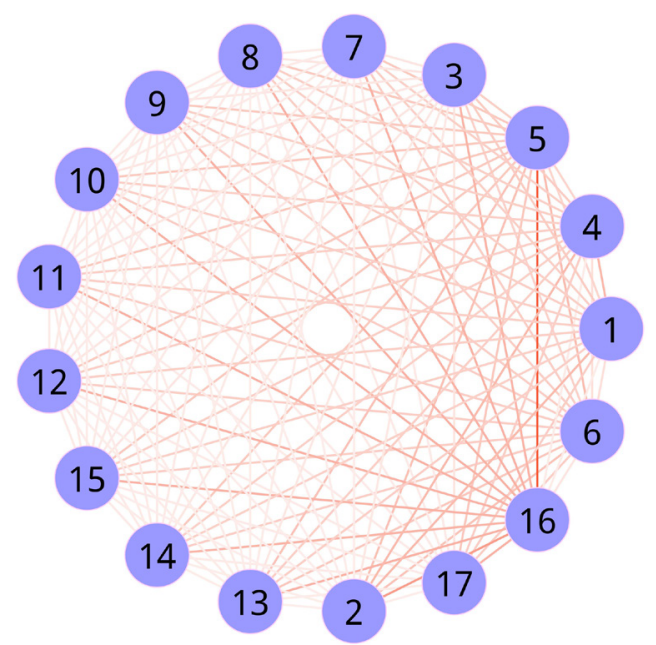

Figure 3. Co-expression network based on 17 pathways. Nodes represent pathways, and edges represent pathway interactions. Deeper color denotes greater interaction (based on weighted values).

By accessing topological centrality analysis for the pathway network, we found that four kinds of centralities (degree, betweenness, closeness, and stress) were similar for the 17 pathways, suggesting that these pathways possessed similar topological properties.

\section{DISCUSSION}

In this paper, we identified 17 significant pathways in HBV-related HCC using pathway crosstalk and network. Interestingly, 9 of the 17 pathways were correlated to PI3K, 7 were signaling related pathways, and regulation of FZD by ubiquitination participated in crosstalk with larger weighted values.

Netrin-1, a diffusible, laminin-related protein, has been identified as neuronal guidance cues during nervous system development, and mainly interacts with receptors deleted in 
colorectal cancer (DCC) and uncoordinated-5-homolog (UNC5H) (Mehlen and Furne, 2005). Moreover, netrin-1 signaling enhances extracellular adenosine signaling (Poth et al., 2013). Netrin-1 overexpression has recently been described in several types of human cancers, such as colorectal cancer (Ko et al., 2014), lung cancer (Delloye-Bourgeois et al., 2009a), and neuroblastoma (Delloye-Bourgeois et al., 2009b). In liver cancer, netrin-1 level was found to be significantly increased in the circulation. Inhibition of netrin-1 receptor results in downregulation of netrin-1-induced apoptosis in tumor cells, and leads to tumor regression (Ramesh et al., 2011). Han et al. (2015) reported that netrin-1 promoted cell migration and invasion by downregulating the expression of blood vessel epicardial substance in HCC. It had been demonstrated that netrin-1-activated downstream signaling pathways induce epithelial-mesenchymal transition with subsequent production of multiple inflammatory mediators, which in turn promotes cancer invasion in hypoxic HCC cells (Yan et al., 2014). Therefore, we hypothesized that Netrin-1 signaling may be a potential biomarker for diagnosis and treatment of HBV-related HCC.

Our pathway analysis revealed that PI3K (phosphatidylinositol 3-kinase) played a key role in the 17 pathways, especially in the PI-3K cascade and PI3K/AKT-related pathways. Dysregulation of the PI3K signaling pathway was believed to exert a potential oncogenic effect in HCC (Dituri et al., 2012). Four PI-3K cascades, FGFR2, FGFR3 and FGFR4, which were members of the fibroblast growth factor receptor (FGFR) family, were explored in our study. It was reported that FGFRs are presented on endothelial cells, and provide critical signaling pathways for HCC metastasis (Presta et al., 2005; Huynh et al., 2008). Schmidt et al. (2016) revealed that the classification system may help identify HCC patients who are most likely to benefit FGFR inhibition. Furthermore, combined FGFR/mTOR treatment showed significant inhibitory effects on signaling and motility in HCC (Scheller et al., 2015). Inhibition of the $\mathrm{PI} 3 \mathrm{~K} / \mathrm{AKT} / \mathrm{mTOR}$ pathway was also shown to be beneficial for suppression of angiogenesis in HCC (Jung et al., 2012), and provided insight into promising strategies for targeted therapies (Fang et al., 2012). Liu et al. (2009) demonstrated that PI3K/AKT signaling is dysregulated in HCC, which suggests that it may be a critical target for therapeutic designs. It may also play an important part in chemokine ligand 5 (CCL5)-mediated migration and invasion of Huh7 cells (Bai et al., 2014). Additionally, activation of PI3K/AKT also compromised the anti-tumor activity of rapamycin in $\mathrm{HCC}$ (Li et al., 2012). In this study, PI3K/AKT activation and PI3K/ AKT signaling were shown to be significant pathways in HCC; this was in agreement with previously published results.

We found that the gene expression profile downloaded from the ArrayExpress database was endemic by HBV to some extent. We also identified that antiviral mechanisms by IFN-stimulated genes correlated closely with HBV. IFN- $\alpha$-mediated epigenetic repression of HBV cccDNA transcriptional activity may therefore assist in the development of novel effective therapeutics (Belloni et al., 2012). This was supported by studies which reported that modulation of IFN-stimulated genes resulted in an antiviral response in target cells aimed at limiting both viral replication and spreading (Sadler and Williams, 2008).

In conclusion, we identified 17 significant pathways in HBV-related HCC based on pathway interaction analysis. These pathways may be potential biomarkers, and give insights into treatment and detection of HBV-related HCC.

\section{Conflicts of interest}

The authors declare no conflict of interest. 


\section{REFERENCES}

Abbasi A, Hossain L and Leydesdorff L (2012). Betweenness centrality as a driver of preferential attachment in the evolution of research collaboration networks. J. Informetrics 6: 403-412. http://dx.doi.org/10.1016/j.joi.2012.01.002

Ahn T, Lee E, Huh N and Park T (2014). Personalized identification of altered pathways in cancer using accumulated normal tissue data. Bioinformatics 30: i422-i429. http://dx.doi.org/10.1093/bioinformatics/btu449

Aoki T, Kokudo N, Matsuyama Y, Izumi N, et al.; Liver Cancer Study Group of Japan (2014). Prognostic impact of spontaneous tumor rupture in patients with hepatocellular carcinoma: an analysis of 1160 cases from a nationwide survey. Ann. Surg. 259: 532-542.http://dx.doi.org/10.1097/SLA.0b013e31828846de

Arzumanyan A, Reis HM and Feitelson MA (2013). Pathogenic mechanisms in HBV- and HCV-associated hepatocellular carcinoma. Nat. Rev. Cancer 13: 123-135. http://dx.doi.org/10.1038/nrc3449

Bai H, Weng Y, Bai S, Jiang Y, et al. (2014). CCL5 secreted from bone marrow stromal cells stimulates the migration and invasion of Huh7 hepatocellular carcinoma cells via the PI3K-Akt pathway. Int. J. Oncol. 45: 333-343.

Belloni L, Allweiss L, Guerrieri F, Pediconi N, et al. (2012). IFN- $\alpha$ inhibits HBV transcription and replication in cell culture and in humanized mice by targeting the epigenetic regulation of the nuclear cccDNA minichromosome. $J$. Clin. Invest. 122: 529-537. http://dx.doi.org/10.1172/JCI58847

Burchard J, Zhang C, Liu AM, Poon RT, et al. (2010). microRNA-122 as a regulator of mitochondrial metabolic gene network in hepatocellular carcinoma. Mol. Syst. Biol. 6: 402. http://dx.doi.org/10.1038/msb.2010.58

Croft D, O'Kelly G, Wu G, Haw R, et al. (2011). Reactome: a database of reactions, pathways and biological processes. Nucleic Acids Res. 39: D691-D697.http://dx.doi.org/10.1093/nar/gkq1018

Delloye-Bourgeois C, Brambilla E, Coissieux MM, Guenebeaud C, et al. (2009a). Interference with netrin-1 and tumor cell death in non-small cell lung cancer. J. Natl. Cancer Inst. 101: 237-247. http://dx.doi.org/10.1093/jnci/djn491

Delloye-Bourgeois C, Fitamant J, Paradisi A, Cappellen D, et al. (2009b). Netrin-1 acts as a survival factor for aggressive neuroblastoma. J. Exp. Med. 206: 833-847. http://dx.doi.org/10.1084/jem.20082299

Diao J, Pantua H, Ngu H, Komuves L, et al. (2012). Hepatitis C virus induces epidermal growth factor receptor activation via CD81 binding for viral internalization and entry. J. Virol. 86: 10935-10949. http://dx.doi.org/10.1128/JVI.00750-12

Dituri F, Mazzocca A, Lupo L, Edling CE, et al. (2012). PI3K class IB controls the cell cycle checkpoint promoting cell proliferation in hepatocellular carcinoma. Int. J. Cancer 130: 2505-2513. http://dx.doi.org/10.1002/ijc.26319

Dweep H and Gretz N (2015). miRWalk2.0: a comprehensive atlas of microRNA-target interactions. Nat. Methods 12: 697.http://dx.doi.org/10.1038/nmeth.3485

Dweep H, Sticht C, Pandey P and Gretz N (2011). miRWalk - database: prediction of possible miRNA binding sites by "walking" the genes of three genomes. J. Biomed. Inform. 44: 839-847.http://dx.doi.org/10.1016/j.jbi.2011.05.002

El-Serag HB (2012). Epidemiology of viral hepatitis and hepatocellular carcinoma. Gastroenterology 142: 1264-1273.e1. http://dx.doi.org/10.1053/j.gastro.2011.12.061

Fang Y, Xue JL, Shen Q, Chen J, et al. (2012). MicroRNA-7 inhibits tumor growth and metastasis by targeting the phosphoinositide 3-kinase/Akt pathway in hepatocellular carcinoma. Hepatology 55: 1852-1862. http://dx.doi. org $/ 10.1002 /$ hep. 25576

Han P, Fu Y, Liu J, Wang Y, et al. (2015). Netrin-1 promotes cell migration and invasion by down-regulation of BVES expression in human hepatocellular carcinoma. Am. J. Cancer Res. 5: 1396-1409.

Haythornthwaite C (1996). Social network analysis: an approach and technique for the study of information exchange. Libr. Inf. Sci. Res. 18: 323-342. http://dx.doi.org/10.1016/S0740-8188(96)90003-1

Huynh H, Ngo VC, Fargnoli J, Ayers M, et al. (2008). Brivanib alaninate, a dual inhibitor of vascular endothelial growth factor receptor and fibroblast growth factor receptor tyrosine kinases, induces growth inhibition in mouse models of human hepatocellular carcinoma. Clin. Cancer Res. 14: 6146-6153.http://dx.doi.org/10.1158/1078-0432.CCR-08-0509

Jiang YF, He B, Li NP, Ma J, et al. (2011). The oncogenic role of NS5A of hepatitis C virus is mediated by up-regulation of survivin gene expression in the hepatocellular cell through p53 and NF-кB pathways. Cell Biol. Int. 35: 1225-1232. http://dx.doi.org/10.1042/CBI20110102

Jung KH, Choi MJ, Hong S, Lee H, et al. (2012). HS-116, a novel phosphatidylinositol 3-kinase inhibitor induces apoptosis and suppresses angiogenesis of hepatocellular carcinoma through inhibition of the PI3K/AKT/mTOR pathway. Cancer Lett. 316: 187-195. http://dx.doi.org/10.1016/j.canlet.2011.10.037

Kaseb AO, Xiao L, Naguib R, El-Shikh W, et al. (2013). Abstract C26: Development and validation of a scoring system using insulin-like growth factor to assess hepatic reserve in hepatocellular carcinoma. Mol. Cancer Ther. 12: C26. http://dx.doi.org/10.1158/1535-7163.TARG-13-C26

Ko SY, Blatch GL and Dass CR (2014). Netrin-1 as a potential target for metastatic cancer: focus on colorectal cancer. Cancer Metastasis Rev. 33: 101-113.http://dx.doi.org/10.1007/s10555-013-9459-z 
Li J and Tibshirani R (2013). Finding consistent patterns: a nonparametric approach for identifying differential expression in RNA-Seq data. Stat. Methods Med. Res. 22: 519-536.http://dx.doi.org/10.1177/0962280211428386

Li QL, Gu FM, Wang Z, Jiang JH, et al. (2012). Activation of PI3K/AKT and MAPK pathway through a PDGFR $\beta$ dependent feedback loop is involved in rapamycin resistance in hepatocellular carcinoma. PLoS One 7: e33379. http://dx.doi.org/10.1371/journal.pone.0033379

Liu P, Cheng H, Roberts TM and Zhao JJ (2009). Targeting the phosphoinositide 3-kinase pathway in cancer. Nat. Rev. Drug Discov. 8: 627-644. http://dx.doi.org/10.1038/nrd2926

Llovet JM and Bruix J (2008). Molecular targeted therapies in hepatocellular carcinoma. Hepatology 48: 1312-1327. http://dx.doi.org/10.1002/hep.22506

Mehlen P and Furne C (2005). Netrin-1: when a neuronal guidance cue turns out to be a regulator of tumorigenesis. Cell. Mol. Life Sci. 62: 2599-2616. http://dx.doi.org/10.1007/s00018-005-5191-3

Myers L and Sirois MJ (2006). Spearman correlation coefficients, differences between. In: Encyclopedia of statistical sciences (Kotz S, Read CB, Balakrishnan N and Vidakovic B, eds.). Johnson Wiley \& Sons, Inc., New York, 12.

Pan J, Lian Z, Wallett S and Feitelson MA (2007). The hepatitis B x antigen effector, URG7, blocks tumour necrosis factor alpha-mediated apoptosis by activation of phosphoinositol 3-kinase and beta-catenin. J. Gen. Virol. 88: 3275-3285. http://dx.doi.org/10.1099/vir.0.83214-0

Poth JM, Brodsky K, Ehrentraut H, Grenz A, et al. (2013). Transcriptional control of adenosine signaling by hypoxiainducible transcription factors during ischemic or inflammatory disease. J. Mol. Med. 91: 183-193. http://dx.doi. org/10.1007/s00109-012-0988-7

Presta M, Dell'Era P, Mitola S, Moroni E, et al. (2005). Fibroblast growth factor/fibroblast growth factor receptor system in angiogenesis. Cytokine Growth Factor Rev. 16: 159-178. http://dx.doi.org/10.1016/j.cytogfr.2005.01.004

Prifti E, Zucker JD, Clément K and Henegar C (2010). Interactional and functional centrality in transcriptional coexpression networks. Bioinformatics 26: 3083-3089. http://dx.doi.org/10.1093/bioinformatics/btq591

Ramesh G, Berg A and Jayakumar C (2011). Plasma netrin-1 is a diagnostic biomarker of human cancers. Biomarkers 16: 172-180. http://dx.doi.org/10.3109/1354750X.2010.541564

Sadler AJ and Williams BR (2008). Interferon-inducible antiviral effectors. Nat. Rev. Immunol. 8: 559-568. http://dx.doi. org/10.1038/nri2314

Scardoni G and Laudanna C (2012). Centralities based analysis of complex networks. In: New frontiers in graph theory (Zhang Y, eds). InTech, Rijeka, 323-348.

Scheller T, Hellerbrand C, Moser C, Schmidt K, et al. (2015). mTOR inhibition improves fibroblast growth factor receptor targeting in hepatocellular carcinoma. Br. J. Cancer 112: 841-850. http://dx.doi.org/10.1038/bjc.2014.638

Schmidt B, Wei L, DePeralta DK, Hoshida Y, et al. (2016). Molecular subclasses of hepatocellular carcinoma predict sensitivity to fibroblast growth factor receptor inhibition. Int. J. Cancer 138: 1494-1505. http://dx.doi.org/10.1002/ ijc. 29893

Scott J (2012). Centrality, peripherality and centralization. In: Social network analysis (Metzler K and Horvai A, eds.). SAGE Publications Ltd., London, 83-98.

Smoot ME, Ono K, Ruscheinski J, Wang PL, et al. (2011). Cytoscape 2.8: new features for data integration and network visualization. Bioinformatics 27: 431-432. http://dx.doi.org/10.1093/bioinformatics/btq675

Yan W, Han P, Zhou Z, Tu W, et al. (2014). Netrin-1 induces epithelial-mesenchymal transition and promotes hepatocellular carcinoma invasiveness. Dig. Dis. Sci. 59: 1213-1221. http://dx.doi.org/10.1007/s10620-013-3016-z 